\title{
Complex Quantum Groups and Their Quantum Enveloping Algebras
}

\author{
Bernhard Drabant $^{1}$, Michael Schlieker ${ }^{2}$, Wolfgang Weich ${ }^{2}$, and Bruno Zumino ${ }^{3}$ \\ 1 Max-Planck-Institut für Physik, Werner-Heisenberg-Institut, Föhringer Ring 6, \\ W-8000 München 40, Federal Republic of Germany \\ 2 Sektion Physik der Universität München, Lehrstuhl Professor Wess, Theresienstrasse 37, \\ W-8000 München 2, Federal Republic of Germany \\ 3 Department of Physics, University of California, Berkeley, California, CA 94720, USA
}

Received November 7, 1991; in revised form March 6, 1992

\begin{abstract}
We construct complexified versions of the quantum groups associated with the Lie algebras of type $A_{n-1}, B_{n}, C_{n}$, and $D_{n}$. Following the ideas of Faddeev, Reshetikhin and Takhtajan we obtain the Hopf algebras of regular functionals $U_{\mathscr{B}}$ on these complexified quantum groups. In the special example $A_{1}$ we derive the $q$-deformed enveloping algebra $U_{q}(s l(2, \mathbb{C}))$. In the limit $q \rightarrow 1$ the undeformed $U(\operatorname{sl}(2, \mathbb{C}))$ is recovered.
\end{abstract}

\section{Introduction}

For quantum groups associated with the Lie algebras $g$ of type $A_{n-1}, B_{n}, C_{n}$, and $D_{n}$ there exist well defined correlations between the quantum group itself and the corresponding $q$-deformed universal enveloping algebra $U_{q}(g)$ [Dri, FRT]. Coming from the quantum group, one can construct the algebra of regular functionals which is shown to be the algebra $U_{q}(g)$ for a certain completion. Though the $q$-deformed Lorentz group already exists in at least two versions [CSSW, PW], there is not yet such a straightforward procedure like in the case of compact Lie groups to derive the corresponding quantized universal enveloping algebra. However this $q$-deformed algebra is the very object of interest since it should be fundamental for the construction of a $q$-deformed relativistic field theory.

In this paper we present the quantized universal enveloping algebra $U_{q}(s l(2, \mathbb{C}))$ of the $q$-deformed Lorentz group $S l_{q}(2, \mathbb{C})$. In Sect. (2) we construct complex quantum groups for the Lie algebras $A_{n-1}, B_{n}, C_{n}$, and $D_{n}$. These are complexifications of the original quantum groups. The algebraic relations can be written in a generalized $R T T$-formulation and the usual determinant or metric relations. Following the ideas of [FRT] this fact is used in Sect. (3) to build up the algebra of regular functionals on the complex quantum groups ${ }^{1}$. The approach in this paper is purely algebraic without

1 The same universal enveloping algebra corresponding to the complex quantum group is constructed by analyzing the algebra of the fundamental bicovariant bicomodule [CW] 
considering the $C^{*}$-structure which has been investigated in [Pod, PW]. In Sect. (4) we derive as a special example the algebra $U_{q}(s l(2, \mathbb{C}))^{2}$. We investigate the limit $q \rightarrow 1$ in Sect. (5) and recover $U(\operatorname{sl}(2, \mathbb{C}))$.

\section{Complexified Quantum Groups}

In the approach of [FRT] the quantum group is a Hopf algebra with comultiplication $\Phi$, counit $e$ and antipode $\kappa$ [Abe], generated by the matrix elements $t^{i}{ }_{j}(i, j=1, \ldots, N$; $N=n$ for $A_{n-1}$ and $N=2 n+1$ for $B_{n}, N=2 n$ for $C_{n}, D_{n}$ ) with the relations

$$
I_{t, t} t_{s t}^{i j}:=\hat{R}_{q}^{i j}{ }_{k l} t^{k}{ }_{s} t_{t}^{l}-t^{i}{ }_{v} t^{j}{ }_{w} \hat{R}_{q}^{v w}{ }_{s t}=0
$$

and

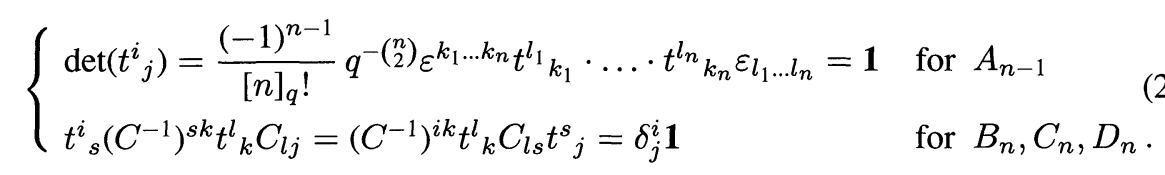

where $\varepsilon_{i_{1} \ldots i_{n}}=(-1)^{n-1} \cdot \varepsilon^{i_{1} \ldots i_{n}}=(-q)^{l(\sigma)},[n]_{q}$ ! is the usual $q$-factorial [CSWW] and $C_{i j}$ is the usual metric [FRT]. The $\hat{R}$-matrices for the respective quantum groups are taken from [FRT] with $q>0$ real.

To find the complexified versions of these quantum groups one has to introduce the complex conjugates $t^{* i}{ }_{j}$ of the generators $t^{i}{ }_{j}$ as additional generators with the complex conjugate versions of the relations (2.1) and (2.2) above [CSWW]. With the definition:

$$
\hat{t}^{i}{ }_{j}:=\left(\kappa\left(t^{j}{ }_{i}\right)\right)^{*}
$$

we get

$$
\begin{gathered}
I_{\hat{t}, \hat{t}_{s t}{ }_{s t}}:=\left(\hat{R}_{q}^{-1}\right)^{i j}{ }_{k l} \hat{t}^{k}{ }_{s} \hat{t}_{t}^{l}-\hat{t}^{i}{ }_{v} \hat{t}^{j}{ }_{w}\left(\hat{R}_{q}^{-1}\right)^{v w}{ }_{s t}=0 \\
\begin{cases}\operatorname{det}\left(\hat{t}^{i}{ }_{j}\right)=\frac{(-1)^{n-1}}{[n]_{q} !} q^{-\left({ }^{n}\right)} \varepsilon^{k_{1} \ldots k_{n}} \hat{t}^{l_{1}}{ }_{k_{1}} \cdot \ldots \cdot \hat{t}^{l_{n}}{ }_{k_{n}} \varepsilon_{l_{1} \ldots l_{n}}=\mathbf{1} & \text { for } A_{n-1} \\
\hat{t}^{i}{ }_{s}\left(C^{-1}\right)^{s k} \hat{t}^{l}{ }_{k} C_{l j}=\left(C^{-1}\right)^{i k} \hat{t}_{k}{ }_{k} C_{l s} \hat{t}^{s}{ }_{j}=\delta_{j}^{i} \mathbf{1} & \text { for } B_{n}, C_{n}, D_{n} .\end{cases}
\end{gathered}
$$

One still has to define commutation relations between the generators $t^{i}{ }_{j}$ and their complex conjugates:

$$
I_{\hat{t}, t_{s t}}^{i j}:=\hat{R}_{q k l}^{i j} \hat{t}^{k}{ }_{s} t_{l}^{l}-t^{i}{ }_{v} \hat{t}^{j}{ }_{w} \hat{R}_{q}^{v w}{ }_{s t}=0 .
$$

With this choice of commutation relations one can identify the function algebra over the unitary group as the quotient $\hat{t}^{i}{ }_{j}=t^{i}{ }_{j}$. There is a second possibility interchanging the role of $t^{i}{ }_{j}$ and $\hat{t}^{i}{ }_{j}$ in (2.6) which is equivalent to the first.

Summarizing we are considering the following quantum group:

$$
\mathscr{b}:=\mathbb{C}\left\langle t^{i}{ }_{j}, \hat{t}^{i}{ }_{j}\right\rangle /\left(I_{t, t_{s t}}{ }^{i j}, I_{\hat{t}, \hat{t}},{ }_{s t}, I_{\hat{t}, t_{s t}}^{i j},(2.2),(2.5)\right) \text {. }
$$

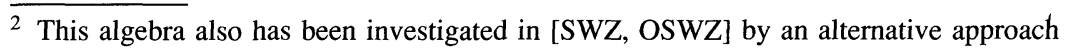


Proposition 1. The algebra Ao becomes a *-Hopf algebra with comultiplication $\Phi$, counit $e$ and antipode $\kappa$ which are defined on the generators through

$$
\begin{aligned}
\Phi\left(t^{i}{ }_{j}\right) & =t^{i}{ }_{k} \otimes t^{k}{ }_{j}, \\
e\left(t^{i}{ }_{j}\right) & =\delta_{j}^{i}, \\
\kappa\left(t^{i}{ }_{j}\right) & = \begin{cases}\frac{q^{-\left(\begin{array}{c}
n \\
2
\end{array}\right)}}{[n-1]_{q} !} \varepsilon^{i k_{1} \ldots k_{n-1}} t^{l_{1}} k_{1} \cdot \ldots \cdot t^{l_{n-1} k_{n-1}} \varepsilon_{l_{1} \ldots l_{n-1} j} & \text { for } A_{n-1} \\
\left(C^{-1}\right)^{i k} t^{l}{ }_{k} C_{l j} & \text { for } B_{n}, C_{n}, D_{n}\end{cases}
\end{aligned}
$$

and

$$
\begin{aligned}
\phi\left(t^{* i}{ }_{j}\right) & =t^{* i}{ }_{k} \otimes t^{* k}{ }_{j}, \\
e\left(t^{* i}{ }_{j}\right) & =\delta_{j}^{i}, \\
\kappa\left(t^{* i}{ }_{j}\right) & =\left(\kappa^{-1}\left(t^{i}{ }_{j}\right)\right)^{*} .
\end{aligned}
$$

It is convenient to introduce an $R T T$-formulation for this complexified quantum group. Set $(I):=(i, \bar{i}), \bar{I}:=(\bar{i}, \overline{\bar{i}})=(\bar{i}, i),(i, \bar{i}=1, \ldots, N)$ and define the $2 N \times 2 N$ matrix,

$$
T_{J}^{I}:=\left(\begin{array}{ll}
t & 0 \\
0 & \hat{t}
\end{array}\right)_{J}^{I} .
$$

Correspondingly one defines the $\mathscr{\mathscr { B }}$-matrix,

$$
\hat{\mathscr{R}}_{q}^{I J} K L:=\left(\begin{array}{cccc}
\alpha_{0} \hat{R}_{q} & 0 & 0 & 0 \\
0 & 0 & \alpha_{1} \hat{R}_{q} & 0 \\
0 & \alpha_{2} \hat{R}_{q}^{-1} & 0 & 0 \\
0 & 0 & 0 & \alpha_{3} \hat{R}_{q}^{-1}
\end{array}\right)
$$

with $\alpha_{i} \in \mathbb{C}$.

Then the relations (2.1), (2.4), and (2.6) can be written in compact form as

$$
\hat{\mathscr{R}}_{q}^{I J}{ }_{K L} T^{K}{ }_{R} T^{L}{ }_{S}=T^{I}{ }_{V} T_{W}^{J} \hat{\mathscr{R}}_{q}^{V W}{ }_{R S},
$$

and $\mathscr{\mathscr { B }}_{q}$ fulfills the Yang-Baxter-Equation:

$$
\left(\mathbf{E} \otimes \mathscr{\mathscr { B }}_{q}\right)\left(\hat{\mathscr{B}}_{q} \otimes \mathbf{E}\right)\left(\mathbf{E} \otimes \hat{\mathscr{B}}_{q}\right)=\left(\hat{\mathscr{B}}_{q} \otimes \mathbf{E}\right)\left(\mathbf{E} \otimes \hat{\mathscr{B}}_{q}\right)\left(\hat{\mathscr{B}}_{q} \otimes \mathbf{E}\right)
$$

with $\mathbf{E}_{J}^{I}=\delta_{J}^{I}$.

There are three further possibilities for the choice of the $\mathscr{\mathscr { B }}_{q}$-matrix which we disregard here, since one of them yields equivalent results and the others do not admit a simple involution on the algebra of regular functionals.

\section{The Algebra of Regular Functionals}

The dual space $\mathscr{A}^{*}$ of the Hopf algebra $\mathscr{A}$ is an algebra with the convolution product. One can introduce an antimultiplication involution "†" on $\mathscr{A}^{*}$ : For $f \in \mathscr{b}^{*}$ one sets

$$
\forall a \in \mathscr{A}: f^{\dagger}(a):=\overline{f\left(\kappa^{-1}\left(a^{*}\right)\right)} .
$$

In the following we are working mostly with the multiplicative involution "-":

$$
\bar{f}:=f^{\dagger} \circ \kappa^{-1} \text {. }
$$


It is also possible to consider an involution where $\kappa^{-1}$ is replaced by $\kappa$ in (3.1) and (3.2). Since $\kappa\left(\left(\kappa\left(a^{*}\right)\right)^{*}\right)=\kappa^{-1}\left(\left(\kappa^{-1}\left(a^{*}\right)\right)^{*}\right)=a \forall a \in \mathscr{A}$ the multiplicative involutions coincide for both cases. This is also true for the antimultiplicative ones for $q \rightarrow 1$. We now construct the algebra of regular functionals on $\mathscr{A}$. We define functionals $L^{ \pm I} \in \mathscr{b}^{*}$ through their action on the generators of $\mathscr{A}$ :

$$
\begin{aligned}
L_{J}^{ \pm I}(1) & :=\delta_{J}^{I}, \\
L_{J}^{ \pm I}\left(T^{k}\right) & :=\mathscr{\mathscr { R }}_{q}^{ \pm 1 I K}{ }_{L J}
\end{aligned}
$$

and their comultiplication

$$
\forall a, b \in \mathscr{A}: L_{J}^{ \pm I}(a b)=L_{K}^{ \pm I}(a) L_{J}^{ \pm K}(b) .
$$

This definition is compatible with the algebra relations in $\mathscr{A}$ and it holds

\section{Proposition 2.}

$$
\begin{aligned}
& L^{ \pm i}{ }_{\bar{j}}=L^{ \pm \bar{j}}=0 \quad \forall i, \bar{j}, \\
& \mathscr{\mathscr { R }}_{q}^{J I} L K L^{ \pm K}{ }_{V} L^{ \pm L}{ }_{W}=L^{ \pm I}{ }_{A} L^{ \pm J}{ }_{B} \hat{\mathscr{R}}_{q}^{B A}{ }_{W V} \text {, } \\
& \hat{\mathscr{R}}_{q}^{J I} L K{ }^{+K}{ }_{V} L^{-L}{ }_{W}=L^{-I}{ }_{A} L^{+J}{ }_{B} \hat{\mathscr{B}}_{q}^{B A}{ }_{W V} \text {. }
\end{aligned}
$$

The equations (2.2) and (2.5) partly determine the coefficients $\alpha_{i}$ in Eq. (2.11):

Proposition 3. For $A_{n-1}$ one has

$$
\left(\alpha_{0}\right)^{-n}=\left(\alpha_{1}\right)^{-n}=\left(\alpha_{2}\right)^{n}=\left(\alpha_{3}\right)^{n}=q .
$$

In the cases of $B_{n}, C_{n}, D_{n}$, one gets

$$
\left(\alpha_{0}\right)^{2}=\left(\alpha_{1}\right)^{2}=\left(\alpha_{2}\right)^{2}=\left(\alpha_{3}\right)^{2}=1 .
$$

Definition. The algebra $U_{\mathscr{R}}$ of regular functionals on $\mathscr{A}$ is the unital algebra generated by $\left\{L^{ \pm I}\right\}$.

Proposition 4. The algebra $U_{\mathscr{R}}$ becomes a bialgebra with comultiplication $\Delta: U_{\mathscr{R}} \rightarrow$ $U_{\mathscr{B}} \otimes U_{\mathscr{B}}$ and counit $\varepsilon: U_{\mathscr{B}} \rightarrow \mathbb{C}$ through the definitions

$$
\begin{aligned}
\Delta\left(L_{J}^{ \pm I}\right) & :=L_{K}^{ \pm I} \otimes L_{J}^{ \pm K} \\
\varepsilon\left(L_{J}^{ \pm I}\right) & :=\delta_{J}^{I}
\end{aligned}
$$

on the generators of $U_{\mathscr{R}}$.

Consider now the map $\tilde{S}: \mathscr{b}^{*} \rightarrow \mathscr{C}^{*}$ defined by

$$
\widetilde{S}:=\cdot \circ \kappa
$$

With this definition we get the following

\section{Proposition 5.}

$$
\tilde{S}\left(U_{\mathscr{B}}\right)=U_{\mathscr{B}}
$$


and

$$
\tilde{S}\left(L^{ \pm i}\right)= \begin{cases}\frac{q^{-\left(\begin{array}{c}
n \\
2
\end{array}\right)}}{[n-1]_{q} !} \varepsilon^{k_{n-1} \ldots k_{1} i} L^{ \pm l_{1}} k_{1} \ldots L^{ \pm l_{n-1} k_{n-1}} \varepsilon_{j l_{n-1} \ldots l_{1}} & \text { for } A_{n-1} \\
\left(C^{-1}\right)^{k i} L^{ \pm l}{ }_{k} C_{j l} & \text { for } B_{n}, C_{n}, D_{n}\end{cases}
$$

$\tilde{S}\left(L^{ \pm \bar{j}} \bar{j}\right)= \begin{cases}\frac{q^{-\left(\begin{array}{c}n \\ 2\end{array}\right)}}{[n-1]_{q} !} \varepsilon^{\bar{k}_{n-1} \ldots \bar{k}_{1} \bar{i}} L^{ \pm \bar{l}_{1}} \bar{k}_{1} \ldots L^{ \pm \bar{l}_{n-1} \bar{k}_{n-1} \varepsilon_{\bar{j} \bar{l}_{n-1} \ldots \bar{l}_{1}}} & \text { for } A_{n-1} \\ \left(C^{-1}\right)^{\bar{k} \bar{i}} L^{ \pm \bar{l}} C_{\bar{k}} C_{\bar{j} \bar{l}} & \text { for } B_{n}, C_{n}, D_{n} .\end{cases}$

it Consequently the algebra $U_{\mathscr{R}}$ becomes a Hopf algebra with antipode $S:=\tilde{S}_{\mid U_{\mathscr{R}}}$. And it holds

$$
L_{J}^{ \pm I} S\left(L_{K}^{ \pm I}\right)=\delta_{K}^{I} e .
$$

Proposition 6. The involution on the generators of $U_{\mathscr{B}}$ is

$$
\overline{L^{ \pm J}}=L_{\bar{J}}^{ \pm \bar{I}}
$$

if

$$
\overline{\alpha_{0}} \cdot \alpha_{3}=1
$$

and

$$
\overline{\alpha_{2}} \cdot \alpha_{1}=1 \text {. }
$$

With the involution " $\dagger$ " $U_{\mathscr{B}}$ becomes a $*$-Hopf algebra. Nevertheless the coefficients $\alpha_{i}$ are not yet completely fixed. For further calculations we introduce the so-called root-of-unity-homomorphisms $e_{r, s}$ which are elements of $\mathscr{A}^{*}$ and are defined multiplicatively on the generators of $\mathscr{t}$ as follows:

$$
\begin{aligned}
e_{r, s}(1) & :=1, \\
e_{r, s}\left(t^{a}{ }_{b}\right) & :=e^{2 \pi i \cdot r / \theta} \cdot \delta_{b}^{a}, \\
e_{r, s}\left(\hat{t}_{\bar{b}}^{\bar{a}_{\bar{b}}}\right) & :=e^{2 \pi i \cdot s / \theta} \cdot \delta_{\bar{b}}^{\bar{a}},
\end{aligned}
$$

where $r, s \in \mathbb{Z}, \Theta:=\left\{\begin{array}{ll}n & \text { for } A_{n-1} \\ 2 & \text { for } B_{n}, C_{n}, D_{n}\end{array}\right.$.

One can easily check the following

Proposition 7. 1. $e_{r, s}$ is a well defined algebra homomorphism,

2. $e_{0,0}=\left(e_{r, s}\right)^{\Theta}=e$,

3. $e_{l, k} \cdot e_{m, n}=e_{l+m, k+n}$,

4. $\left[e_{r, s}, f\right]=0 \forall f \in \mathscr{G}^{*}$,

5. $\overline{e_{r, s}}=e_{s, r}$.

Using the special form of the $\mathscr{\mathscr { B }}_{q}$-matrix and the form of the matrices $\hat{R}_{q}$ for $A_{n-1}, B_{n}, C_{n}$, or $D_{n}$, we get

Proposition 8. 1. $L^{+i}{ }_{j}$ is upper-triangular, $L^{+{ }_{i}}{ }_{j}$ is lower-triangular.

2. $L^{+i}{ }_{i} \cdot L^{+\bar{i}_{i}}=L^{+\bar{i}_{\bar{i}}} \cdot L^{+i}{ }_{i}=e_{l, l}$, where $\alpha_{0} \cdot \alpha_{2}=\alpha_{1} \cdot \alpha_{3}=e^{2 \pi i \cdot l / \Theta}$.

3. $L^{-\bar{i}}{ }_{\bar{j}}=L_{j}^{-i} \cdot e_{r,-r}$, where $\alpha_{0} \cdot \alpha_{1}^{-1}=\left(\alpha_{2} \cdot \alpha_{3}{ }^{-1}\right)^{*}=e^{2 \pi i \cdot r / \Theta}$.

4. $\left[L^{+i}{ }_{i}, L_{j}^{+j}\right]=\left[L^{+\bar{i}_{i}}, L_{\bar{j}}^{+\bar{j}}\right]=0$.

5. $L^{+1}{ }_{1} \cdot \ldots \cdot L^{+N}{ }_{N}=L^{+\overline{1}_{1}} \cdot \ldots \cdot L^{+\bar{N}}{ }_{\bar{N}}=e$ for $A_{n-1}, C_{n}, D_{n}$ and $\left(L_{1}^{+1} \cdot \ldots \cdot L_{N}^{+N}\right)^{2}=\left(L^{+\overline{1}_{\overline{1}}} \cdot \ldots \cdot L^{+\bar{N}}{ }_{\bar{N}}\right)^{2}=$ e for $B_{n}$. 


\section{The Hopf Algebra $U_{q}(s l(2, \mathbf{C}))$}

To illustrate the above developed formalism we now investigate the easiest example, that is the Hopf algebra $U_{q}(s l(2, \mathbb{C}))$ with the additional choice $\alpha_{0}=\alpha_{1}$. The other possibility, $\alpha_{0}=-\alpha_{1}$, would provide the additional algebra homomorphism $e_{1,1}$ in $U_{\mathscr{B}}$. We do not consider this case in this paper. As a consequence of these restrictions we get $\alpha_{0} \cdot \alpha_{2}=\alpha_{0} \cdot\left(\alpha_{1}\right)^{-1}=1$ and thus the equations in Proposition 8 only contain $e_{0,0}=e$. Therefore in the case $A_{1}$ we only have to consider the unit $e$ and the generators

$$
L^{+1}{ }_{1}, L^{+1}{ }_{2}, L^{+\overline{2}}{ }_{1}, L^{-1}{ }_{1}, L^{-1}, L^{-2}{ }_{1}, L^{-2}{ }_{2},\left(L^{+1}{ }_{1}\right)^{-1} \text {. }
$$

For further considerations we define the element

$$
\Delta:=L_{2}^{-1} \cdot L_{1}^{-2} \in U_{\mathscr{B}} .
$$

Proposition 9. 1. $\left\{\Delta^{n} \mid n \in \mathbb{N}^{0}\right\}$ is a linearly independent set in $\mathscr{A}^{*}$.

2. $\Delta^{n}=0$ for monomials $t^{g_{1}} \hat{t}^{g_{2}}$ with $\min \left(g_{1}, g_{2}\right)<n$.

Property 2 of Proposition 9 allows us to handle power series in $\mathscr{A}^{*}$ of the form

$$
\begin{aligned}
& \Lambda_{1}^{1}=L_{1}^{-1}\left(e+\sum_{n=1}^{\infty} \alpha_{n} \Delta^{n}\right), \\
& \Lambda_{2}^{2}=L^{-2}\left(e+\sum_{n=1}^{\infty} \beta_{n} \Delta^{n}\right),
\end{aligned}
$$

where $\alpha_{n}, \beta_{n}$ are arbitrary complex numbers. Because of this fact, property 1 of Proposition 9 and (3.12) we obtain

Proposition 10. $L^{-1}{ }_{1}$ is invertible and $\left(L^{-1}{ }_{1}\right)^{-1}=L^{-2}{ }_{2}\left(e+\sum_{n=1}^{\infty}(-q)^{-n} \Delta^{n}\right)$ is an
element of a certain minimal extension of $U_{\mathscr{B}}$.

Consequently there remain six essential generators since $L^{-2}{ }_{2}$ can now be expressed through $\Delta$ and $\left(L^{-1}{ }_{1}\right)^{-1}$. Using (3.5) and (3.12) we get the following algebra relations:

$$
\begin{gathered}
{\left[L^{-1}{ }_{2}, L^{-2}{ }_{1}\right]=0 \quad\left[L^{-1}{ }_{1}, L^{+1}{ }_{1}\right]=0, \quad\left[L^{+1}{ }_{2}, L^{-1}{ }_{2}\right]=\left[L^{-2}{ }_{1}, L^{+\overline{2}}{ }_{\overline{1}}\right]=0,} \\
{\left[L^{-2}{ }_{1}, L^{+1}{ }_{2}\right]=\left(q-q^{-1}\right)\left\{\left(L^{+1}{ }_{1}\right)^{-1} L^{-1}{ }_{1}-\left(L^{-1}{ }_{1}\right)^{-1}\left(e+q^{-1} \Delta\right) L^{+1}{ }_{1}\right\},} \\
{\left[L^{-1}{ }_{2}, L^{+\overline{2}}{ }_{\overline{1}}\right]=\left(q-q^{-1}\right)\left\{L^{-1}{ }_{1} L^{+1}{ }_{1}-\left(L^{+1}{ }_{1}\right)\left(L^{-1}{ }_{1}\right)^{-1}\left(e+q^{-1} \Delta\right)\right\}} \\
{\left[L^{+1}{ }_{2}, L^{+\overline{2}}{ }_{\overline{1}}\right]=\left(q-q^{-1}\right)\left\{\left(L^{+1}{ }_{1}\right)^{2}-\left(L^{+1}{ }_{1}\right)^{-2}\right\},} \\
L^{ \pm 1}{ }_{1} L^{ \pm 1}{ }_{2}=q^{-1} L^{ \pm 1}{ }_{2} L^{ \pm 1}{ }_{1}, L^{-1}{ }_{1} L^{-2}{ }_{1}=q^{-1} L^{-2}{ }_{1} L^{-1}{ }_{1}, L^{-1}{ }_{2} L^{+1}{ }_{1}=q L^{+1}{ }_{1} L^{-1}{ }_{2}, \\
L^{-2}{ }_{1} L^{+1}{ }_{1}=q^{-1} L^{+1}{ }_{1} L^{-2}{ }_{1}, \quad L^{+\overline{2}}{ }_{1} L^{+1}{ }_{1}=q^{-1} L^{+1}{ }_{1} L^{+\overline{2}}{ }_{\overline{1}}, \\
L^{-1}{ }_{1} L^{+\overline{2}}{ }_{1}-q L^{+\overline{2}}{ }_{1} L^{-1}{ }_{1}=\left(q^{-1}-q\right) L^{-2}{ }_{1}\left(L^{+1}{ }_{1}\right)^{-1}, \\
L^{-1}{ }_{1} L^{+1}{ }_{2}-q L^{+1}{ }_{2} L^{-1}{ }_{1}=\left(q^{-1}-q\right) L^{-1}{ }_{2} L^{+1}{ }_{1} .
\end{gathered}
$$

In the next step we make an ansatz similar to [FRT] with $H_{i}, X_{i}^{ \pm} ; i=1,2$. We set

$$
\begin{aligned}
& L^{+1}{ }_{1}=\exp \left(h / 2 H_{1}\right), \quad L^{-1}{ }_{1}=\exp \left(h / 2 H_{2}\right), \quad L^{+1}{ }_{2}=-\left(q-q^{-1}\right) X_{1}^{-}, \\
& L^{+\overline{2}}{ }_{\overline{1}}=\left(q-q^{-1}\right) X_{1}^{+}, \quad L^{-2}{ }_{1}=\left(q-q^{-1}\right) X_{2}^{-}, \quad L^{-1}{ }_{2}=-\left(q-q^{-1}\right) X_{2}^{+} \text {, }
\end{aligned}
$$

where $q=e^{h}$. 
The equations (4.4) and (4.5) yield the following algebra relations:

$$
\begin{gathered}
{\left[H_{1}, H_{2}\right]=\left[X_{1}^{ \pm}, X_{2}^{\mp}\right]=\left[X_{2}^{+}, X_{2}^{-}\right]=0, \quad\left[H_{1}, X_{1}^{ \pm}\right]= \pm 2 X_{1}^{ \pm}} \\
{\left[H_{1}, X_{2}^{ \pm}\right]=\mp 2 X_{2}^{ \pm}, \quad\left[H_{2}, X_{2}^{ \pm}\right]=-2 X_{2}^{ \pm}} \\
{\left[H_{2}, X_{1}^{ \pm}\right]=2 X_{1}^{ \pm}-4 X_{2}^{\mp} \exp \left(\mp h / 2\left(H_{1} \pm H_{2}\right)\right)} \\
{\left[X_{1}^{+}, X_{1}^{-}\right]=\frac{\exp \left(h H_{1}\right)-\exp \left(-h H_{1}\right)}{\left(q-q^{-1}\right)}} \\
{\left[X_{1}^{ \pm}, X_{2}^{ \pm}\right]=\frac{\exp \left( \pm h / 2\left(H_{1} \pm H_{2}\right)\right)-\exp \left(\mp h / 2\left(H_{1} \pm H_{2}\right)\right)}{\left(q-q^{-1}\right)}} \\
+\left(1-q^{2}\right) \exp \left(\mp h / 2\left(H_{1} \pm H_{2}\right)\right) X_{2}^{+} X_{2}^{-} .
\end{gathered}
$$

Coming from $H_{i}, X_{i}^{ \pm}$one can argue that this algebra is a certain completion of $U_{\mathscr{B}}$ and a $*$-Hopf algebra with coproduct $\Delta$,

$$
\begin{gathered}
\Delta\left(X_{1}^{ \pm}\right)=X_{1}^{ \pm} \otimes \exp \left(-h / 2 H_{1}\right)+\exp \left(h / 2 H_{1}\right) \otimes X_{1}^{ \pm}, \\
\Delta\left(X_{2}^{+}\right)=X_{2}^{+} \otimes \exp \left(-h / 2 H_{2}\right)\left(e-q^{-2} \mathscr{D}\right)+\exp \left(h / 2 H_{2}\right) \otimes X_{2}^{+}, \\
\Delta\left(X_{2}^{-}\right)=X_{2}^{-} \otimes \exp \left(h / 2 H_{2}\right)+\exp \left(-h / 2 H_{2}\right)\left(e-q^{-2} \mathscr{D}\right) \otimes X_{2}^{-}, \\
\Delta\left(H_{1}\right)=H_{1} \otimes e+e \otimes H_{1}, \\
\Delta\left(H_{2}\right)=\frac{2}{h} \sum_{k=1}^{\infty} \frac{1}{k}(-1)^{k-1}\left(\exp \left(h / 2\left(H_{2} \otimes e+e \otimes H_{2}\right)\right)\right. \\
\left.-\left(q-q^{-1}\right)^{2} X_{2}^{+} \otimes X_{2}^{-}+-e \otimes e\right)^{k},
\end{gathered}
$$

antipode $S$

$$
\begin{gathered}
S\left(X_{1}^{ \pm}\right)=-\exp \left(-h / 2 H_{1}\right) X_{1}^{ \pm} \exp \left(h / 2 H_{1}\right), \\
S\left(X_{2}^{ \pm}\right)=-\exp \left(\mp h / 2 H_{2}\right) X_{2}^{ \pm} \exp \left( \pm h / 2 H_{2}\right), \\
S\left(H_{1}\right)=-H_{1}, \\
S\left(H_{2}\right)=\frac{2}{h} \sum_{k=1}^{\infty} \frac{1}{k}(-1)^{k-1}\left(\exp \left(-h / 2 H_{2}\right)\left(e-q^{-2} \mathscr{D}\right)-e\right)^{k},
\end{gathered}
$$

and counit $\varepsilon$

$$
\varepsilon\left(H_{1}\right)=\varepsilon\left(H_{2}\right)=0, \quad \varepsilon\left(X_{1}^{ \pm}\right)=\varepsilon\left(X_{2}^{ \pm}\right)=0,
$$

where $\mathscr{D}:=q\left(q-q^{-1}\right)^{2} X_{2}^{+} X_{2}^{-}$.

As a formal power series in $h$ the generators $H_{1}$ and $H_{2}$ are well defined and unique [Ogi].

\section{The Limit $q \rightarrow 1$}

We investigate the limit $q \rightarrow 1$ for the algebra relations (4.6). A short computation yields

$$
\begin{gathered}
{\left[H_{1}, H_{2}\right]=\left[X_{1}^{ \pm}, X_{2}^{\mp}\right]=\left[X_{2}^{+}, X_{2}^{-}\right]=0, \quad\left[H_{1}, X_{1}^{ \pm}\right]= \pm 2 X_{1}^{ \pm},} \\
{\left[H_{1}, X_{2}^{ \pm}\right]=\mp 2 X_{2}^{ \pm}, \quad\left[H_{2}, X_{2}^{ \pm}\right]=-2 X_{2}^{ \pm},} \\
{\left[H_{2}, X_{1}^{ \pm}\right]=2 X_{1}^{ \pm}-4 X_{2}^{\mp}, \quad\left[X_{1}^{+}, X_{1}^{-}\right]=H_{1},} \\
{\left[X_{2}^{-}, X_{1}^{-}\right]=1 / 2\left(H_{1}-H_{2}\right), \quad\left[X_{1}^{+}, X_{2}^{+}\right]=1 / 2\left(H_{1}+H_{2}\right) .}
\end{gathered}
$$


To recover the usual $U(\operatorname{sl}(2, \mathbb{C}))$-structure, we transform the Lie algebra (5.1) linearly

$$
\begin{array}{ccc}
\hat{H}_{1}:=1 / 2\left(H_{1}-H_{2}\right), & \hat{H}_{2}:=1 / 2\left(H_{1}+H_{2}\right), & \hat{X}_{1}^{+}:=X_{2}^{-}, \\
\hat{X}_{1}^{-}:=\left(X_{1}^{-}-X_{2}^{+}\right), & \hat{X}_{2}^{+}:=\left(X_{1}^{+}-X_{2}^{-}\right), & \hat{X}_{2}^{-}:=X_{2}^{+} .
\end{array}
$$

With (5.1) and (5.2) we get the relations

$$
\begin{aligned}
& {\left[\hat{H}_{i}, \hat{X}_{i}^{ \pm}\right]= \pm 2 \hat{X}_{\imath}^{ \pm}, \quad\left[\hat{X}_{i}^{+}, \hat{X}_{i}^{-}\right]=\hat{H}_{i}, \quad\left[\hat{H}_{1}, \hat{H}_{2}\right]=0} \\
& {\left[\hat{H}_{1}, \hat{X}_{2}^{ \pm}\right]=\left[\hat{H}_{2}, \hat{X}_{1}^{ \pm}\right]=0, \quad\left[\hat{X}_{1}^{ \pm}, \hat{X}_{2}^{ \pm}\right]=\left[\hat{X}_{1}^{ \pm}, \hat{X}_{2}^{\mp}\right]=0}
\end{aligned}
$$

and the involution

$$
\hat{H}_{1}^{\dagger}=\hat{H}_{2}, \quad\left(\hat{X}_{1}^{ \pm}\right)^{\dagger}=\hat{X}_{2}^{\mp} .
$$

Considering comultiplication and antipode in this limit one recovers the universal enveloping algebra of $\operatorname{sl}(2, \mathbb{C})$.

\section{Concluding Remarks}

Apart from our work there are three further papers which deal with the same object [SWZ, OSWZ, CW] and, closely related, with the $q$-deformed Poincaré algebra [LNRT]. In [SWZ, OSWZ] a $q$-deformed version of the Lorentz algebra is derived via linear representations of the algebra on the complex spinor quantum plane. This yields a 7-generator algebra with additional parameter [SWZ]. This algebra can be found in the enveloping algebra of a 6-generator formulation of $U_{q}(s l(2, \mathbb{C}))$ [OSWZ].

Using the algebra $U_{\mathscr{B}}$ a differential calculus is developed in [CW] (see footnote in Sect.(1)). This algebra of differential operators is another formulation of the algebra $U_{q}(s l(2, \mathbb{C}))$.

Another approach uses the $q$-generalization of the adjoint representation of Lie groups to derive the analogous of the linear functionals in [Wor, CSWW, Jur] which correspond to the left invariant vector fields on the Lie group in the limit $q \rightarrow 1$. This is now under investigation [CDSWZ].

Acknowledgements. It is a pleasure for us to thank Julius Wess for drawing our attention to this problem. We would also like to thank R. Dick and O. Ogievetsky for valuable discussions.

\section{References}

[Abe] Abe, E.: Hopf algebras. Cambridge tracts in Mathematics, Vol. 74. Cambridge: Cambridge University Press (1980)

[CDSWZ] Chryssomalakos, C., Drabant, B., Schlieker, M., Weich, W., Zumino, B.: Vector fields on complex quantum groups. Commun. Math. Phys. 147, 635-646 (1992)

[CSSW] Carow-Watamura, U. Schlieker, M., Scholl, M., Watamura, S.: Tensor representation of the quantum group $S L_{q}(2, \mathbb{C})$ and quantum Minkowski space. Z. Phys. C 48, 159-165 (1990);

Carow-Watamura, U. Schlieker, M., Scholl, M., Watamura, S.: A quantum Lorentz group. Int. J. Mod. Phys. A 6, No. 17, 3081-3108 (1991)

[CSWW] Carow-Watamura, U. Schlieker, M., Watamura, S., Weich, W.: Bicovariant differential calculus on quamtum groups $S U_{q}(N)$ and $S O_{q}(N)$. Commun. Math. Phys. 142, 605-641 (1991)

[CW] Carow-Watamura, U. Watamura, S.: Tokhu preprint (1991)

[Dri] Drinfel'd V.G.: Quantum groups. In: Proceedings of the International Congress of Mathematicians, Berkeley 1986, pp. 798-820. Berkeley 1986 
[FRT] 'Faddeev, L.D., Reshetikhin, N.Yu., Takhtajan, L.A.: Quantization of Lie groups and Lie algebras. In: Algebra Analysis 1, 178 (1987)

[Jur] Jurčo, B.: Differential calculus on quantized simple Lie groups. Lett. math. Phys. 22, 177 (1991)

[LNRT] Lukierski, J., Nowicki, A., Ruegg, H., Tolstoy, V.N.: Geneva preprints VGVA-DPT 1991/02-710, VGVA-DPT 1991/08-740 to appear in Phys. Lett. B (1991)

[Ogi] Ogievetsky, O.: Private communication

[OSWZ] Ogievetsky, O., Schmidke, W.B., Wess, J., Zumino, B.: Six generator $q$-deformed Lorentz algebra. MPI/Ph/91-51 (1991)

[Pod] Podlés, P.: Complex quantum groups. Preprint RIMS 754 (1991)

[PW] Podlés, P., Woronowicz, S.L.: Quantum deformation of Lorentz group. Commun. Math. Phys. 130, 381-431 (1990)

[SWZ] Schmidke, W.B., Wess, J., Zumino, B.: A $q$-deformed Lorentz algebra. Preprint MPI$\mathrm{Ph} / 91-15$ (1991)

[Wor] Woronowicz, S.L.: Differential calculus on compact matrix pseudogroups (quantum groups). Commun. Math. Phys. 122, 125-170 (1989)

Communicated by A. Connes 
